\title{
Current Recommendations for the Management of Cancer-Associated Venous Thromboembolism
}

\author{
Katalin Makó \\ Department of Internal Medicine, "George Emil Palade" University of Medicine, Pharmacy, Science and Technology, Târgu \\ Mureș, Romania
}

\section{ABSTRACT}

Cancer-associated thrombosis (CAT) is a major cause of death in oncological patients. The mechanisms of thrombogenesis in cancer patients are not fully established, and it seems to be multifactorial in origin. Also, several risk factors for venous thromboembolism (VTE) are present in these patients such as tumor site, stage, histology of cancer, chemotherapy, surgery, and immobilization. Anticoagulant treatment in CAT is challenging because of high bleeding risk during treatment and recurrence of VTE. Current major guidelines recommend low molecular weight heparins (LMWHs) for early and long-term treatment of VTE in cancer patients. In the past years, direct oral anticoagulants (DOACs) are recommended as potential treatment option for VTE and have recently been proposed as a new option for treating CAT. This manuscript will give a short overview of risk factors involved in the development of CAT and a summary on the recent recommendations and guidelines for treatment of VTE in patients with malignancies, discussing also some special clinical situations (e.g. renal impairment, catheter-related thrombosis, and thrombocytopenia).

Keywords: cancer-associated thrombosis, low molecular weight heparins, direct oral anticoagulants

\section{ARTICLE HISTORY}

Received: February 7, 2021

Accepted: April 15, 2021

\section{CORRESPONDENCE}

\section{Katalin Makó}

Str. Gheorghe Marinescu nr. 38 540139 Târgu Mureș, Romania

Tel: +40265215551

E-mail: makokatalin@yahoo.com

\section{INTRODUCTION}

Malignancy and oncological therapies are well recognized risk factors for developing venous thromboembolism (VTE), the absolute risk mainly depending on tumor type, stage, and treatment with antineoplastic agents. ${ }^{1}$ Annually, approximately $0.5 \%$ of cancer patients develop VTE compared with a rate of incidence of $0.1 \%$ in the non-cancer population. ${ }^{2}$ Cancer-associated thrombosis (CAT) is a life-threatening condition and is the second most prevalent cause of mortality in oncological patients, the development of VTE increasing the likelihood of death 2- to 6-fold and the probability of fatal pulmonary embolism (PE) 3-fold. ${ }^{1-5}$ Approximately $5-20 \%$ of patients with cancer will develop VTE, the highest rate of thrombosis occurring within the first few months after the diagnosis. Moreover, patients with metastatic cancer are at higher risk for VTE. Therefore, optimal prevention, treatment, and follow-up of these patients is crucial. Patients diagnosed with VTE always require anticoagulant therapy. However, the management of CAT is challenging due to possible bleeding complications and recurrence of deep vein thrombosis (DVT) despite optimal anticoagulation therapy. This manuscript will give a short overview of risk factors involved in the development of CAT and a summary on the recent recommendations and guidelines for treatment of VTE in patients with malignancies, discussing also some special clinical situations (e.g. renal impairment, catheter-related thrombosis, and thrombocytopenia). 


\section{CLINICAL FORMS OF CANCER- RELATED THROMBOSIS}

Thrombotic complications in cancer can manifest as venous or arterial thrombosis, or disseminated intravascular coagulation (DIC). All components of the Virchow triad (hypercoagulable state, endothelial injury, circulatory stasis) can be present in oncological patients. Cancer induces procoagulant changes, thus increasing the levels of proteins with procoagulant activity and reducing the natural anticoagulant mechanisms. In addition, chemotherapy may induce endothelial injury, while surgical interventions, immobilization, and local compression are associated with venous stasis and endothelial dysfunction. ${ }^{5}$

The most common clinical manifestation of CAT is venous thromboembolism. In the National Comprehensive Cancer Network (NCCN) guidelines, the definition of VTE includes DVT, pulmonary embolism (PE), superficial vein thrombosis (SVT), and splanchnic vein thrombosis (SPVT). ${ }^{6}$

Arterial thrombosis is not very common in patients with cancer compared to the prevalence of VTE. However, multiple studies and case reports suggest that patients with cancer present an increased risk of arterial thrombosis. In a iarge retrospective study Navi et al. investigated the relation between cancer and the risk of arterial thrombosis in patients with active cancer, which was found to be $4.7 \%$ at 6 months, compared to $2.2 \%$ in the control group. ${ }^{7}$

DIC and thrombotic microangiopathy (TMA) are rare, yet severe complications in oncological patients. In DIC, an intravascular activation of the coagulation cascade occurs with a subsequent propensity for both thrombosis and bleeding produced by intravascular fibrin deposition, consumption of thrombocytes, and coagulation factors. Generally, the clinical presentation and evolution of cancer-related DIC is less fulminant than DIC associated with other etiologies, such as sepsis or trauma, and may be the first clinical symptom of cancer. ${ }^{8}$ TMA may occur as thrombotic thrombocytopenic purpura (TTP) or hemolytic uremic syndrome (HUS), with ischemic end-organ damage (brain, kidney) produced by disseminated occlusive microvascular thrombotic occlusion and thrombocytopenia. It can also be a manifestation of cancer or a complication of cancer therapy. ${ }^{9}$

\section{RISK ASSESSMENT MODELS FOR THROMBOSIS IN PATIENTS WITH CANCER}

Recently, a large number of risk factors and biomarkers have been described as predictive for VTE. These risk factors can be cancer-associated, including tumor site, and the type and/or the stage of cancer. In addition, there are other risk factors associated with the patient such as

TABLE 1. The validated risk assessment models for evaluate the risk of venous thromboembolism and the variables used in different models

\begin{tabular}{|c|c|c|c|c|}
\hline & $\begin{array}{c}\text { Khorana } \\
\text { score }^{11}\end{array}$ & $\begin{array}{l}\text { Oncothromb } \\
\text { Tic-Onco } \\
\text { score } \\
\text { extended }^{15}\end{array}$ & $\begin{array}{l}\text { Compass } \\
\text { CAT score }{ }^{16}\end{array}$ & $\begin{array}{c}\text { Pabinger } \\
\text { clinical } \\
\text { prediction } \\
\text { model }^{18}\end{array}$ \\
\hline Type of tumor & $x$ & $x$ & - & $x$ \\
\hline Cancer stage & - & $x$ & $x$ & - \\
\hline Time since cancer diagnosis & - & - & $x$ & - \\
\hline Genetic risk score & - & $x$ & - & - \\
\hline Personal history of VTE & - & - & $x$ & - \\
\hline VTE family history & - & - & $x$ & - \\
\hline Body mass index $\left(\mathrm{BMI}>35 \mathrm{~kg} / \mathrm{m}^{2}\right)$ & $x$ & $x$ & - & - \\
\hline Presence of cardiovascular risk factors & - & - & $x$ & - \\
\hline Recent hospitalization for acute medical illness & - & - & $x$ & - \\
\hline Anemia (hemoglobin $<10 \mathrm{~g} / \mathrm{dL}$ ) & $x$ & - & - & - \\
\hline Leukocytosis (white blood cell count $>11 \times 10^{9} / \mathrm{L}$ ) & $x$ & - & - & - \\
\hline Thrombocytosis (platelet count $\geq 350 \times 10^{9} / \mathrm{L}$ ) & $x$ & - & - & - \\
\hline d-Dimer $>1.44 \mathrm{~g} / \mathrm{L}$ & - & - & - & $x$ \\
\hline Soluble $\mathrm{P}$-selectin $>53.1 \mathrm{~g} / \mathrm{L}$ & - & - & - & - \\
\hline Anthracycline or antihormonal therapy & - & - & $x$ & - \\
\hline Central venous catheter & - & - & $x$ & - \\
\hline
\end{tabular}


age, sex, race, genetic risk score, immobility, previous VTE, obesity, and other medical comorbidities. An important category of risk factors is represented by oncological treatment. Chemotherapy and anti-angiogenic treatment are known as having prothrombotic effects. Various serum biomarkers, including anemia, increased leukocyte count, thrombocyte count, D-dimer levels, P-selectin and tissue factor, have also been associated with an increased risk of CAT. ${ }^{10}$ A large number of risk assessment models (RAMs) for VTE risk prediction have been proposed: the Khorana score,${ }^{11}$ the Vienna CATS score, ${ }^{12}$ the PROTECHT score, ${ }^{13}$ the CONKO score, ${ }^{14}$ the Oncothromb Tic-Onco extended score,${ }^{15}$ the Compass CAT score,${ }^{16}$ the Oncotev score, ${ }^{17}$ and the Pabinger clinical prediction model. ${ }^{18}$ The clinical effectiveness of these RAMs in particular subtypes of tumors are still a matter of debate. Validated risk assessment models for CAT are presented in Table 1.

The risk of developing CAT in different types of cancer has been evaluated in a large number of studies but it is difficult to compare them directly. In a general analysis of patients with malignancies, the highest rates of CAT have been found in patients with pancreas, brain, gastric, renal, lung, uterus, and ovary tumors, as well as in hematological malignancies. ${ }^{10,17}$ The advanced stage of oncological disease represents also a major risk factor for CAT. In a population-based study that included 389 cancer patients with newly diagnosed VTE, Blom et al. reported that the presence of distant metastases represents a higher risk of CAT. ${ }^{19}$

The histological subtype of cancer can also predict the risk of CAT in some types of malignancies. The Vienna Cancer and Thrombosis Study has compared the importance of local, regional, and distant cancer stages on the occurrence of CAT. The study found a significantly more increased risk of thrombosis in case of regional and/or metastatic disease compared to patients with early, localized stages of cancer. ${ }^{2,18}$

The time after diagnostic confirmation may also provide important predictive information for VTE in oncological patients. Naess et al. have published the results of a retrospective study that included over 200,000 oncological patients. Their results revealed a risk for developing CAT of $3.3 \%$ within the first year, which decreased to $0.8 \%$ after 2 years from diagnosis. ${ }^{20}$

\section{DEMOGRAPHIC DATA, COMORBIDITIES, PRIOR DVT AS RISK FACTORS FOR CAT}

Age may also have an important impact on the development of VTE, as older subjects with various oncological disorders are at a higher risk for venous or arterial thrombotic events. In a retrospective cohort study published by Khorana et al., patients aged over 65 years with cancer presented a significantly higher likelihood for developing CAT compared to younger persons. ${ }^{21}$

Retrospective studies which have evaluated the effect of gender on the risk of CAT have shown that female patients present higher risk for venous thrombotic events, compared to males who have a higher propensity for developing arterial site thrombosis. ${ }^{21}$

Obesity is a known major risk factor for VTE in the general population, and it is applicable for oncological patients as well. A body mass index (BMI) of $\geq 35 \mathrm{~kg} / \mathrm{m}^{2}$ is one of the five variables in the Khorana risk prediction model, ${ }^{11}$ and a BMI $\geq 25 \mathrm{~kg} / \mathrm{m}^{2}$ is a variable included in the TiC-Onco score. ${ }^{15} \mathrm{~A}$ large number of studies have described a strong relationship between the incidence of CAT and the presence various medical comorbidities such as heart disease, renal impairment, respiratory disorders. ${ }^{2,21}$ In a retrospective review, acute infections were associated with a higher risk of CAT, especially for hospitalized cancer patients. ${ }^{22}$

Prior episodes of thrombosis or PE represent major risk factors for recurrent thrombosis both in general and oncological populations. In a prospective study on patients with cancer undergoing surgery, it was shown that patients with previous thrombosis presented a significantly higher rate of new or recurrent VTE (OR $=6.0 ; 95 \%$ CI 2.1 to 16.8$).{ }^{23}$

\section{SERUM BIOMARKERS AS RISK MARKERS FOR VTE}

Several laboratory biomarkers have been proven predictive for VTE in cancer patients, ranging from simple complete blood count components to novel biomarkers.

Elevated pre-chemotherapy thrombocyte number, defined as a platelet count of $350,000 / \mathrm{mm}^{3}$, was identified as an important risk factor for CAT. In the Vienna Cancer and Thrombosis Study (CATS), 665 patients with solid tumors were included. The one-year probability of CAT in this analysis was $34.3 \%$ in patients with a platelet number above the 95 th percentile $\left(443 \times 10^{9} / \mathrm{L}\right)$ compared to $5.9 \%$ in those with a thrombocyte count below this value. Thrombocytosis, soluble P-selectin, and surgery were significantly associated with the risk of CAT after multivariate analysis was performed, along with age, leucocyte count, chemotherapy, and radiotherapy. ${ }^{24}$

A leukocyte count $>11,000 / \mu \mathrm{L}$ before initiating chemotherapy was described as a significant and independent risk factor for VTE. ${ }^{24}$ In a prospective analysis that in- 
cluded 4,405 ambulatory cancer patients for whom chemotherapy was initiated, there was a significantly higher rate of thrombotic events in patients with leukocytosis ( 25 out of $561,4.5 \%$ ), compared to subjects with normal leucocyte count ( 68 out of 3,860, 1.8\%) (p<0.0001). ${ }^{25}$ Significantly higher rates of VTE were also found in patients who had persistent leukocytosis after the first chemotherapy cycle (3.0\%) compared to those with resolved baseline leukocytosis $(1.7 \%)$ or patients without leukocytosis throughout the whole course of chemotherapy $(1.2 \%)(\mathrm{p}=0.03) .{ }^{25}$

$\mathrm{D}$-dimer is an indicator for activation of the coagulation cascade and is generally elevated in patients with cancer. This particular biomarker may be used as a predictive indicator for cancer metastasis and progression. ${ }^{26}$ Higher levels of D-dimer can also predict recurrent thrombosis in oncological patients. ${ }^{27}$ Evaluation of serum D-dimer levels is recommended in patients that are scheduled for chemotherapy, as elevated pre-treatment values have been correlated with treatment-related CAT. ${ }^{28,29}$ The level of D-dimer measured before initiation of chemotherapy has been described as an independent risk factor for thrombosis in chemo-naïve patients with pancreatic adenocarcinoma, ${ }^{30}$ or in patients with advanced gastric cancer undergoing palliative chemotherapy. ${ }^{31} \mathrm{~A}$ metaanalysis performed by $\mathrm{Wu}$ et al. aimed to investigate the importance of plasma D-dimer levels in patients with ovarian malignancies. The analysis demonstrated that elevated levels of D-dimer indicate an increased risk of developing VTE in patients with ovarian cancer $(\mathrm{OR}=4.068$, $95 \%$ CI 2.423 to 6.829$).^{32}$

$\mathrm{P}$-selectin is a cell adhesion molecule known as an independent risk factor for VTE. In a prospective study, Ay et al. included 687 cancer patients (lung, gastrointestinal, breast, pancreas, renal, prostate, and brain malignancies). ${ }^{33}$ After multivariate analysis, a higher P-selectin level (cutoff level of $53.1 \mathrm{ng} / \mathrm{mL}$, 75th percentile of study population) was an independent predictor for thrombosis, after adjustment for age, gender, surgical intervention, and chemo- and radiotherapy was made. The cumulative probability of developing CAT at six months after study inclusion was $11.9 \%$ in cases with a P-selectin level above and $3.7 \%$ in patients with $\mathrm{P}$-selectin below the 75th percentile $(\mathrm{p}=0.002){ }^{33}$

\section{VTE RELATED TO CANCER TREATMENT}

Chemotherapy is one of the most important factors associated with an increased risk of thrombosis, especially in the first 3-6 months of treatment, which present 6-7 times higher rates of chemotherapy-associated thrombotic events. $2,21,28,34,35$ The mechanism of chemotherapyinduced DVT in patients with cancer is plurifactorial and is produced by the inappropriate activation of hemostasis, partly due to increased procoagulant activity or to a dysfunction of the anticoagulant protein $\mathrm{C}$ and protein $\mathrm{S}$ pathway. ${ }^{34}$ Substantial risk of VTE has been reported in patients undergoing platinum-based chemotherapy (cisplatin, oxaliplatin). ${ }^{36,37}$ Cancer patients receiving immunosuppressive or cytotoxic chemotherapy, such as l-asparaginase, thalidomide, lenalidomide, or tamoxifen, also present an elevated risk for VTE. ${ }^{38}$

VTE is a frequent complication in oncological patients undergoing surgical interventions. Surgery increases the risk of postoperative DVT and fatal PE more than 2-3fold compared with similar surgical interventions on patients without malignancy..$^{38}$ If a patient with active cancer is hospitalized for an acute medical illness, the incidence of VTE is also elevated. ${ }^{2}$ Central venous catheters are also important risk factors for VTE development, and in case of catheter-related thrombosis serious complications may occur, including fatal PE. The true incidence of catheterrelated thrombosis is not known due to its unspecific symptoms. However, it is estimated to present an incidence ranging between 5 and $30 \%{ }^{39}$

\section{THERAPEUTIC MANAGEMENT OF CANCER-ASSOCIATED THROMBOSIS}

The management of oncological patients with DVT is often challenging for clinicians. These patients present a 2 -fold greater risk for major bleeding and 3-fold higher risk of DVT recurrence compared to patients without cancer..$^{40}$ Therapeutic anticoagulation is the key component in the management of CAT. Current recommendations on initial treatment and long-term therapy of cancer-related VTE, thromboprophylaxis in hospitalized cancer patients or in case of cancer surgery, and some special situations (anticoagulation in case of renal impairment, thrombocytopenia) will be discussed below.

Low-molecular-weight heparin (LMWH) alone or in combination with vitamin $\mathrm{K}$ antagonists (VKAs) was the standard anticoagulation therapy for CAT for many years.

The CLOT trial, published in 2003, included 676 patients receiving dalteparin or VKA for a six-month period with 5-7 days of overlap period. Symptomatic recurrent VTE, including PE-related death, occurred in $7.0 \%$ of patients randomized to dalteparin versus $15 \%$ randomized to VKA ( $\mathrm{p}=0.002$ ). However, there were no differences in major bleeding, any bleeding, or death rates. ${ }^{41}$ This study 
confirmed the superiority of LMWH in the long-term anticoagulant therapy of patients with cancer. More than 10 years later, the results of the CATCH trial were published. In this trial, 900 patients were randomized to tinzaparin (175 IU/kg) or warfarin (tinzaparin overlap for 5-10 days) for 6 months. The primary composite endpoint was incidental recurrent proximal DVT and PE, which occurred in $6.9 \%$ of patients receiving tinzaparin and in $10 \%$ of patients from the warfarin treatment $\operatorname{arm}(\mathrm{p}=0.07) .^{42}$ The major rates of bleeding were similar, but a significant reduction in non-major clinically relevant bleeding was observed with tinzaparin. ${ }^{42,43}$ The CANTHANOX, ONCENOX, and LITE trials also showed that LMWHs were superior in prevention of recurrent DVT and PE in patients with cancer. The bleeding profiles were improved or were similar compared to VKAs. $44,45,46$

The recommendation on initial parenteral anticoagulation therapy remained unchanged since 2016. For the first 5-10 days of VTE, anticoagulant therapy with LMWH is recommended, with unfractionated heparin (UFH) and fondaparinux as options for alternative treatment. 47,48

Two Cochrane reviews from the last several years compared LMWH to UFH or fondaparinux. ${ }^{49,50} \mathrm{~A}$ meta-analysis which included 446 cancer patients described a significant reduction in mortality for LMWH compared to UFH (OR $0.53,95 \%$ CI 0.33 to $0.85, \mathrm{p}=0.009) . .^{50}$ In a meta-analysis, Hakoum et al..$^{49}$ showed a decreasing trend in mortality and VTE recurrence at three months with LMWH compared to UFH. Fondaparinux-treated patients did not present significant difference in DVT recurrence for mortality or bleeding. ${ }^{50}$

For newly diagnosed VTE, parenteral anticoagulant therapy with LMWH is preferred over UFH for the initial period ( 5 to 10 days), given that the creatinine clearance is over $30 \mathrm{~mL}$ per minute. ${ }^{48,51} \mathrm{UFH}$ and fondaparinux may be used as alternative therapy to LMWH (when are contraindicated, or not available).

Direct oral anticoagulants (DOACs) as antifactor Xa inhibitors (rivaroxaban, apixaban, edoxaban) and direct thrombin inhibitor (dabigatran) have been approved for the initial, long-term, and extended treatment of DVT..$^{52}$ Several randomized clinical trials and meta-analyses have compared DOACs with LMWH in patients with cancer. ${ }^{53-55}$

Raskob et al. randomly assigned oncological patients with acute symptomatic or incidental DVT to receive either edoxaban (after at least five days of pre-treatment with LMWH) or subcutaneous dalteparin. ${ }^{33}$ The composite outcome of recurrent VTE or major bleeding for oral edoxaban was noninferior to subcutaneous dalteparin (HR, $0.97,95 \%$ CI 0.70 to $1.36, p=0.006$ for non-infe- riority). The recurrence rate of VTE was lower, but major bleeding was more frequent with edoxaban compared to dalteparin. ${ }^{53}$

The SELECT-D trial conducted by Young et al. ${ }^{54}$ randomly assigned patients with active cancer and VTE (a total number of 406 patients, $58 \%$ of whom with metastases) to receive treatment with either rivaroxaban or dalteparin. The primary outcome was the recurrence of VTE over six months, while the safety was the occurrence of major bleeding and clinically relevant non-major bleeding. The 6-month rate of VTE recurrence was $11 \%$ ( $95 \%$ CI $7 \%$ to $16 \%$ ) with dalteparin and $4 \%$ (95\% CI $2 \%$ to $9 \%$ ) with rivaroxaban (HR $0.43,95 \%$ CI 0.19 to 0.99 ) without significant difference in major bleeding rate $(6 \%$ with rivaroxaban vs. $4 \%$ with dalteparin, HR 1.83, 95\% CI 0.68 to 4.96). The clinically relevant non-major bleeding risk was higher with rivaroxaban compared to dalteparin (13\% vs. $4 \%$, HR $3.76,95 \%$ CI 1.63 to 8.69$) .54$

In a meta-analysis of the Hokusai VTE Cancer and SELECT-D trials, DOACs were associated with a lower risk of VTE but the risk of major bleeding was higher compared with LMWH, without any significant difference in mortality. ${ }^{55}$

In the current guidelines, ${ }^{48,51}$ based on evidence from randomized clinical trials, ${ }^{53,54}$ rivaroxaban and edoxaban (after minimum 5 days of parenteral anticoagulation) have been added as treatment options in CAT, in patients without increased risk for gastrointestinal or genitourinary bleeding.

Some important practical issues must be emphasized regarding treatment with DOACs. Firstly, there are differences between DOACs regarding the initiation of anticoagulant treatment in the first days. VTE patients without cancer require 5 days of parenteral anticoagulation with LMWH before starting edoxaban treatment in standard, therapeutic dose.53 For rivaroxaban, LMWH pretreatment is not needed, but in the first 21 days a higher dose is recommended ( $15 \mathrm{mg}$ bid for the first 21 days of anticoagulation, then $20 \mathrm{mg}$ daily). Particular caution for treatment with DOACs is needed in patients with an increased risk for bleeding (active mucosal lesions or unresected mucosal tumors) or other conditions associated with additional hemorrhagic risk (chronic kidney disease, hepatic impairment, gastrointestinal bleeding in history, thrombocytopenia, antiplatelet therapy). In case of DOAC treatment in cancer patients, an important safety consideration is the potential for drug-drug interaction. Inducers and inhibitors of P-glycoprotein interact with edoxaban and rivaroxaban, and rivaroxaban can also interact with potent inhibitors or inducers of cyto- 
chrome P 450 34A.48,51,55 Due to the oral administration of DOACs, their effects can also be influenced by gastrointestinal absorption and tolerability, as well as nausea and vomiting. ${ }^{56}$

\section{GUIDELINE RECOMMENDATIONS FOR VTE ANTICOAGULATION IN CANCER PATIENTS}

\section{Initial (5-10 days) parenteral anticoagulation}

In case of cancer-related VTE, for the initial treatment the drug of choice is LMWH at a body weight-adjusted dose when creatinine clearance is over $30 \mathrm{~mL} / \mathrm{min} .{ }^{48,51,57}$ As a therapeutic alternative, rivaroxaban or edoxaban (after at least 5 days of parenteral anticoagulant therapy) can also be used for patients without high risk of gastrointestinal or genitourinary bleeding or important drug-drug interactions, given a creatinine clearance over $30 \mathrm{~mL} / \mathrm{min} .{ }^{48,51}$ UFH and fondaparinux also can be a therapeutic alternative used for the initial treatment of VTE in cancer patients in case of contraindication of LMWH or DOACs, or if these are not available. . $^{8,51,57}$

As per recent guidelines in case of CAT, thrombolysis can be considered on a case-by-case basis and possible complications, especially the risk of bleeding, must be carefully evaluated. Inferior vena cava filters for initial treatment of VTE in cancer patients may be considered when anticoagulation is contraindicated or in case of recurrence VTE under optimal anticoagulation. ${ }^{48,51}$

\section{Early maintenance (up to 6 months)}

From 2016, the clinical practice guidelines recommend LMWHs at body weight-adjusted dose as the preferred treatment over VKAs for early maintenance therapy (up to 6 months). ${ }^{41-45,47,48,51}$ Since the 2016 guidelines, no new randomized trials have been published comparing LMWH with VKAs.

In recent guidelines, rivaroxaban and edoxaban are also recommended for early maintenance (up to 6 months) treatment of CAT (if creatinine clearance is over $\geq 30 \mathrm{~mL} /$ min) in patients without risk of drug-drug interactions or gastrointestinal absorption impairment. ${ }^{48,51}$ VKAs are considered inferior in this setting, but may be used in case of inaccessibility of LMWH, rivaroxaban, or edoxaban. ${ }^{51}$

Special caution in using DOACs is needed in case of an increased bleeding risk, mainly in patients with gastrointestinal tract (especially upper tract) or urogenital malignancies. Data for other DOACs in early maintenance therapy for cancer-related DVT are needed. The minimum period of using LMWH or DOACs (rivaroxaban, apixaban) in treating patients with malignancies and established VTE should be 6 months. ${ }^{51}$

\section{Long-term (beyond 6 months) treatment}

The scientific data about the benefits and possible risks of anticoagulant treatment beyond 6 months in patients with cancer are limited. The prospective, multicenter DALTECAN trial included 334 patients with newly diagnosed DVT and active cancer receiving subcutaneous dalteparin for 12 months. In total, 109 patients completed the 12-month course of dalteparin therapy. The risk of developing recurrent DVT or major bleeding complications was highest in the first month of treatment and lower in the next 11 months..$^{58}$ The TiCAT trial also evaluated the effects of LMWH (tinzaparin) treatment beyond 6 months in patients with CAT, on 247 patients with established VTE and active cancer. The incidence of major bleeding was $4.9 \%$, and the rate of clinically relevant non-major bleeding (CRNMB) was $0.9 \%$ during the first 6 months and $0.6 \%$ in the next 7 to 12 months $(\mathrm{p}=0.5) .^{59}$

The decision of cessation or continuation of anticoagulation (LMWH, DOACs, or VKAs) must be established considering the risk of bleeding, cancer activity, quality of life, life expectancy, cost of therapy, and also patient preference. The continuation of anticoagulant treatment after the initial 6-month period should established after an individual evaluation of selected active cancer patients, especially those who have metastatic cancer or those on active chemotherapy. . $^{48,51}$

\section{THROMBOPROPHYLAXIS IN PATIENTS WITH ACTIVE CANCER}

\section{PERIOPERATIVE PROPHYLAXIS OF VTE}

DVT is a very frequent and major complication in patients with malignancies undergoing surgery. VTE is the cause of $10 \%$ of perioperative deaths, the risk of a postoperative, fatal PE being 4 times higher compared to the non-cancer population. The risk of DVT depends on a series of factors including the type and stage of tumor, the anesthesia and the type of surgical procedure, comorbidities, age, and period of post-interventional immobilization, as well as previous DVT. ${ }^{23,57,60}$ A large number of randomized trials and meta-analyses have demonstrated the beneficial role of prophylactic anticoagulation (LMWH or UFH) over no thromboprophylaxis or placebo. Bergqvist et al. published 
data of a double-blind, multicenter trial in patients with abdominal or pelvic malignancies, with planned open $\mathrm{cu}-$ rative surgery. The patients were prescribed enoxaparin (40 mg daily) for the first 6 to 10 days and after that were assigned randomly to receive enoxaparin or placebo for another 21 days. The primary efficacy endpoint was the incidence of DVT/PE between days 25 and 31. The rates of VTE were $4.8 \%$ in the enoxaparin group and $12.0 \%$ in the placebo group ( $\mathrm{p}=0.02$ ), the difference persisting at 3 months ( $13.8 \%$ vs. $5.5 \%, p=0.01)$ without significant differences in the rates of bleeding or other complications during the study period. ${ }^{61}$

The risk of VTE persists for several weeks after abdominal or pelvic oncological surgery. Bustos Merlo et al. performed a multicenter, prospective study on data from symptomatic DVT oncological patients. They analyzed 766 patients, out of which $52 \%$ presented VTE. Most VTE cases $(84 \%)$ occurred within the first week after surgery and $38 \%$ after one month. The venous thrombotic event occurred particularly in patients operated for colorectal, gynecologic, and urologic malignancies. ${ }^{62}$

In 2016, Fagarasanu et al. published a review and metaanalysis of 4,807 patients for which thromboprophylaxis was extended, and this led to a decrease in the incidence of all VTE, but there was no difference in the incidence of symptomatic PE vs. major bleeds and all-cause death (RR $=0.44){ }^{63}$

According to guideline recommendations, all patients with cancer undergoing major surgery should receive pharmacological thromboprophylaxis with either LMWH or UFH if there is no contraindication for anticoagulation (active bleeding or high bleeding risk). Mechanical methods are not recommended as the sole treatment strategy unless pharmacological anticoagulation is contraindicated (high risk of bleeding). The combination of pharmacological and mechanical methods may increase efficacy in preventing DVT, especially in high-risk cancer patients. ${ }^{48,51}$

\section{PROPHYLAXIS OF VTE IN ONCOLOGICAL PATIENTS HOSPITALIZED FOR AN ACUTE MEDICAL ILLNESS}

Patients with active malignancy hospitalized with an acute medical condition or with reduced mobility even in the absence of additional pro-thrombotic risk factors should be offered thromboprophylaxis in the absence of contraindications. In these patients, treatment with $\mathrm{LMWH}$, fondaparinux (when creatinine clearance is $\geq 30 \mathrm{~mL} / \mathrm{min}$ ), or with UFH is recommended. DOACs are not recommended routinely in this setting. ${ }^{48,51}$

\section{THROMBOPROPHYLAXIS IN AMBULATORY PATIENTS WITH MALIGNANCIES AND SYSTEMIC CHEMOTHERAPY}

The risk for thrombosis in ambulatory cancer patients with systemic chemotherapy is approximately $5-10 \%$. The risk of VTE depends on the type, stage of cancer, the treatment used, and other patient characteristics. ${ }^{48,64}$ Guideline recommendations state that primary routine pharmacologic thromboprophylaxis is not recommended in ambulatory cancer patients receiving systemic chemotherapy. ${ }^{48,51}$

Several RCTs and meta-analyses performed in ambulatory cancer patients receiving systemic anticancer therapies were published recently, comparing thromboprophylaxis with LMWH, apixaban, or rivaroxaban to no treatment or placebo. In a randomized trial, Khorana et al. have evaluated the benefit of 12 weeks of thromboprophylaxis with dalteparin (5,000 IU daily) in high-risk outpatients with malignancies versus no prophylaxis. ${ }^{65} \mathrm{~A}$ total number of 98 patients were randomized, and DVT occurred in $12 \%$ receiving dalteparin and $21 \%$ with no prophylaxis (HR 0.69, 95\% CI 0.23 to 1.89). There were no differences in major bleeding rates between the groups. ${ }^{65}$ An updated meta-analysis assessed 26 randomized trials comparing any anticoagulant therapy (parenteral or oral) or mechanical intervention to no therapeutic intervention or placebo (a total number of 12,352 patients). ${ }^{64} \mathrm{LMWH}$ significantly decreased the incidence of DVT or PE (RR $0.54,95 \%$ CI 0.38 to 0.75 ) with no significant increase in the risk of bleeding. ${ }^{64}$

In the CASSINI trial, ambulatory patients with malignancies and high thrombotic risk (Khorana score of $\geq 2$ ), were randomized to receive rivaroxaban (10 $\mathrm{mg}$ per die) or placebo daily for 6 months, with screening at every 2 months. ${ }^{66}$ The primary composite endpoint was symptomatic or asymptomatic VTE and VTE-related death. From the 841 randomized patients, $6.0 \%$ presented the primary endpoint from the rivaroxaban group and $8.8 \%$ from the placebo group (HR 0.66, p = 0.10) for an average follow-up period of 180 days. Major bleeding occurred in $2.0 \%$ of patients from the rivaroxaban group and in $1.0 \%$ from the placebo group (HR 1.96, 95\% CI 0.59 to 6.49). ${ }^{66}$

The randomized, placebo-controlled, double-blind AVERT trial ${ }^{67}$ assessed the efficacy and safety of apixaban (2.5 mg bid) for thromboprophylaxis in ambulatory treated patients with malignancies and intermediate to high risk for VTE, who were started on chemotherapy. ${ }^{67}$ The modified intention-to-treat analysis revealed a lower risk of symptomatic and incidental VTE with apixaban vs. placebo ( $4.2 \%$ vs. $10.2 \%, \mathrm{p}<0.001$ ), but an increased risk of major bleeding (3.5\% vs. $1.8 \%$, HR $2.00, \mathrm{p}=0.046) .{ }^{67}$ 
Assessing the risk of VTE is recommended in ambulatory patients with malignancies before the initiation of systemic anticancer therapy, during the treatment, and during the evolution of disease. ${ }^{57}$ Routine primary pharmacologic thromboprophylaxis in outpatient settings during the systemic anticancer therapy should not be offered. ${ }^{48,51}$ For ambulatory patients with malignancies and high thrombotic risk (Khorana score $\geq 2$, prior chemotherapy, locally advanced or metastatic pancreatic cancer) thromboprophylaxis with apixaban, rivaroxaban, or LMWH is recommended, in the presence of an acceptable risk of bleeding. Initiation of anticoagulant therapy, the benefits, side effects, cost and duration of treatment should be discussed with every patient..$^{51}$

\section{THROMBOPROPHYLAXIS AND DVT TREAMENT IN PATIENTS WITH CENTRAL VENOUS CATHETERS}

Central venous catheters (CVCs) are commonly used in oncological diseases, with an increase in the risk for upper limb DVT and subsequent pulmonary embolism. The incidence of symptomatic upper limb DVT related to CVCs ranges between $0.3 \%$ and $28.3 \%$, and $27-66 \%$ of subclinical thrombotic events can be detected by venography. ${ }^{68}$ Risk factors for CVC-associated thrombosis include the type of catheter, placement technique and location, cancer stage, and treatment (chemotherapy, anti-angiogenic therapy, hormone- or radiotherapy). The recent guidelines not recommend routine thromboprophylaxis in patients with CVCs and malignancies. ${ }^{48,51}$ Kahale et al. performed a meta-analysis of 13 studies (3,420 patients with cancer and CVCs) and revealed that LMWH decreased the incidence of clinically manifest catheter-related DVT for a follow-up period of up to 3 months compared to no treatment (RR $0.43,95 \%$ CI 0.22 to 0.81 ) without increase in bleeding complications. ${ }^{69}$ The meta-analysis did not confirm or infirm a beneficial or detrimental effect of low-dose VKA compared with no treatment on mortality, clinically manifest catheter-related VTE, major or minor bleeding. ${ }^{69}$ As result of several studies and one metaanalysis, it is recommended that CVCs should be placed in the right jugular vein with the catheter tip positioned at the right atrium/superior vena cava junction. ${ }^{57,70,71}$

Regarding treatment of thrombosis associated with CVC, the scientific evidence is scarce. In this setting, data derived from recommendations regarding lower limb DVT are used with LMWH is recommended minimum 3 months and indefinite treatment should be considered in case of active cancer and if the catheter is not removed. The catheter should not be removed unless it is no longer needed, it is infected, or there is any contraindication to anticoagulation. ${ }^{57,72}$ Studies with direct comparisons between LMWHs, DOACs, and AVKs in this setting have not been conducted..$^{48}$

\section{THERAPEUTIC MANAGEMENT OF CAT IN SPECIAL SITUATIONS}

\section{INCIDENTAL VTE EVENTS IN CANCER PATIENTS}

Incidental VTE is a frequent problem in patients with cancer, accounting up to half of all thrombotic events. This will probably increase in the future with the large-scale use of modern imaging techniques. The available data on the optimal treatment of incidentally discovered isolated $\mathrm{PE}$ or splanchnic vein thrombosis is scarce. ${ }^{73}$ According to recent guidelines, the therapeutic recommendations for incidental VTE are the same as for symptomatic VTE: anticoagulation with LMWH or DOACs is the standard regimen. In case of isolated, incidental subsegmental PE or splanchnic vein thrombosis the treatment should be individualized. ${ }^{48,51}$

\section{TREATMENT OF VTE RECURRENCE DURING ANTICOAGULATION TREATMENT}

In absence of randomized clinical trials to guide the therapeutic management of recurrent DVT in oncological patients, empirical anticoagulant therapy is the suggested approach. Firstly, treatment compliance and the correct dosage of anticoagulant medication should be controlled, and the probability of thrombocytopenia induced by heparin should be evaluated. If the patient is treated with LMWH, a dose increase or switch to DOAC is recommended. Two retrospective studies support the increase of LMWH dose with $25 \% .74,75$ A switch to DOACs could be a solution in this setting due to the lower rate of recurrence compared to LMWH published in clinical trials. ${ }^{53,54}$ If the patient is treated with VKAs while the DVT recurrence occurs, the therapeutic solution should be the switch to therapeutic doses of LWMH or DOACs. ${ }^{57}$ If recurrence is diagnosed, while on prescribed DOACs, a dose escalation or switch to LMWH should be considered. If there are major contraindications for anticoagulation, or in case of failure of anticoagulant therapy, the insertion of a vena cava filter may be considered.

\section{PATIENTS WITH THROMBOCYTOPENIA}

Anticoagulant treatment for VTE in cancer patients with thrombocytopenia is controversial. According to some 
publications, the administration of low-dose LMWH or temporary discontinuation of anticoagulation in case of severe thrombocytopenia may be safely and effectively used. Other studies suggest anticoagulation with LMWH in a therapeutic dose with transfusion support. Samuelson Bannow et al. performed a systematic review on 121 cancer patients with VTE and thrombocytopenia, in which they reported an increased risk of VTE recurrence (27\%). In $15 \%$ of all patients included in this review, there was an episode of major bleeding, regardless of their treatment strategy. Thus, the authors concluded that there is no superiority of one management strategy over another for the therapeutic management of CAT in patients with thrombocytopenia. ${ }^{76}$

According to current guidelines, in case of established cancer-related VTE with associated thrombocytopenia, the therapeutic doses can be used if the platelet count is $>50,000$ cells/L in patients without manifest bleeding. In patients with a platelet count $<50,000$ cells/L, the therapeutic management and dosage should be individualized. In case of mild thrombocytopenia (platelet count $>80,000$ cells/L) pharmacological thromboprophylaxis can be used, but in case of severe thrombocytopenia (platelet count $<80$,000 cells/L) pharmacological thromboprophylaxis must be individualized and careful monitored..$^{8}$

\section{PATIENTS WITH RENAL FAILURE}

Renal impairment is a frequent challenge in patients with cancer and VTE, being associated with a higher risk of recurrent DVT and also bleeding. Treatment with LMWHs or VKAs may represent a cause of higher bleeding risk complications in renal failure. Available prospective data on CAT in patients with renal insufficiency are limited, the recommendations for anticoagulant therapy for DVT in patients with cancer and renal insufficiency being unchanged since the 2016 guidelines. ${ }^{47} \mathrm{~A}$ recent sub-analysis from the CLOT trial ${ }^{41}$ in a subgroup of patients with impaired renal function (creatinine clearance $<60 \mathrm{~mL} / \mathrm{min}$ ) showed that treatment with dalteparin did not produce an increase in the bleeding risk compared to VKA, while efficacy remained unchanged. Therefore, dalteparin confers a significantly reduced risk of recurrent VTE. ${ }^{41}$

According to current guidelines, in case of severe renal impairment (creatinine clearance $<30 \mathrm{~mL} / \mathrm{min}$ ) the suggested treatment of established DVT is UFH followed by early VKAs (possibly from day one) or LMWH adjusted to anti-Xa level. In these patients, an external compression device can also be applied. In patients with severe renal failure (creatinine clearance $<30 \mathrm{~mL} / \mathrm{min}$ ) pharmacologi- cal prophylaxis could be considered on a case-by-case basis..$^{48}$

\section{CONCLUSIONS}

Cancer-associated thrombosis is a life-threatening condition and is the second most prevalent cause of death in cancer patients. Oncological patients undergoing major surgery or systemic treatment, or those hospitalized for an acute medical illness are at an especially increased risk for VTE. Therefore, optimal prevention, treatment, and follow-up of these patients is crucial. The diagnosis of VTE always requires anticoagulant therapy. However, the management of CAT is challenging due to possible bleeding complications and recurrence of DVT despite optimal anticoagulation. Evidence-based guidelines from professional organizations provide clinicians with recommendations for anticoagulation strategies for the specific management of cancer patients. Adherence to clinical practice guidelines improves our ability to effectively and safely treat and prevent thromboembolic complications in patients with malignancies.

\section{CONFLICT OF INTEREST}

Nothing to declare.

\section{REFERENCES}

1. Timp JF, Braekkan SK, Versteeg HH, et al. Epidemiology of cancer- associated venous thrombosis. Blood. 2013;122:17121723. doi: 10.1182/blood-2013-04-460121.

2. Abdol Razak NB, Jones G, Bhandari M, et al. Cancerassociated thrombosis: An overview of mechanisms, risk factors, and treatment. Cancers. 2018;10:380. doi: 10.3390/ cancers10100380.

3. Chew HK, Wun $\mathrm{T}$, Harvey $\mathrm{D}$, et al. Incidence of venous thromboembolism and its effect on survival among patients with common cancers. Arch Intern Med. 2006;66:458-464. doi: 10.1001/archinte.166.4.458

4. Sorensen HT, Mellemkjaer L, OlsenJH, etal.Prognosis of cancers associated with venous thromboembolism. N Engl J Med. 2000;343:1846-1850. doi: 10.1056/NEJM200012213432504.

5. Wójtowicz M, Rękas-Wójcik A, Lipa A, et al. Thrombotic complications - prevention and treatment of venous thromboembolism in cancer patients. Journal of Pre-Clinical and Clinical Research. 2012;6:84-87.

6. Streiff MB, Holmstrom B, Angelini D, et al. NCCN Guidelines Insights: Cancer-Associated Venous Thromboembolic Disease, Version 2.2018. J Natl Compr Canc Netw. 2018;16:1289-1303. doi: 10.6004/jnccn.2018.0084.

7. Navi BB, Reiner AS, Kamel $\mathrm{H}$, et al. Risk of Arterial Thromboembolism in Patients With Cancer. J Am Coll Cardiol. 2017;70:926-938. doi: 10.1016/j.jacc.2017.06.047. 
8. Levi M. Management of cancer-associated disseminated intravascular coagulation. Thrombo Res. 2016;140:S66-S70. doi: 10.1016/S0049-3848(16)30101-3.

9. Govind Babu K, Bhat GR. Cancer-associated thrombotic microangiopathy. Ecancermedicalscience. 2016;10:649. doi: 10.3332/ecancer.2016.649.

10. Connolly GC, Khorana AA. Emerging risk stratification approaches to cancer-associated thrombosis: risk factors, biomarkers and a risk score. Thromb Res. 2010;125:S1-S7. doi: 10.1016/S0049-3848(10)00227-6.

11. Khorana AA, Kuderer NM, Culakova E, et al. Development and validation of a predictive model for chemotherapy-associated thrombosis. Blood. 2008;111:4902-4907. doi: 10.1182/ blood-2007-10-116327.

12. Ay C, Dunkler D, Marosi C, et al. Prediction of venous thromboembolism in cancer patients. Blood. 2010;116:53775382. doi: 10.1182/blood-2010-02-270116.

13. Verso M, Agnelli G, Barni S, et al. A modified Khorana risk assessment score for venous thromboembolism in cancer patients receiving chemotherapy: the Protecht score. Intern Emerg Med. 2012;7:291-292. doi: 10.1007/s11739-012-0784-y.

14. Pelzer U, Sinn M, Stieler J, et al. Primary pharmacological prevention of thromboembolic events in ambulatory patients with advanced pancreatic cancer treated with chemotherapy? Dtsch Med Wochenschr. 2013;138:2084-2088. doi: 10.1055/s0033-1349608.

15. Muñoz Martín AJ, Ortega I, Font C, et al. Multivariable clinicalgenetic risk model for predicting venous thromboembolic events in patients with cancer. Br J Cancer. 2018;118:10561061. doi: 10.1038/s41416-018-0027-8.

16. Khorana AA, DeSancho MT, Liebman H, et al. Prediction and Prevention of Cancer-Associated Thromboembolism. Oncologist. 2021;26:e2-e7. doi: 10.1002/onco.13569.

17. Stein PD, Beemath A, Meyers FA, Skaf E, Sanchez J, Olson $\mathrm{RE}$. Incidence of venous thromboembolism in patients hospitalized with cancer. Amer J Med. 2006;119:60-68. doi: 10.1016/j.amjmed.2005.06.058.

18. Dickmann B, Ahlbrecht J, Pabinger I. Regional lymph node metastases are a strong risk factor for venous thromboembolism: Results from the Vienna Cancer and Thrombosis Study. Haematologica. 2013;98:1309-1314. doi: 10.3324/haematol.2012.073338.

19. Blom JW, Doggen CJ, Osanto S, Rosendaal FR. Malignancies, prothrombotic mutations, and the risk of venous thrombosis. JAMA. 2005;293:715-722. doi: 10.1001/jama.293.6.715.

20. Næss IA, Christiansen SC, Romundstad P, et al. Incidence and mortality of venous thrombosis: A population-based study. J Thromb Haemost. 2007;5:692-699. doi: 10.1111/j.15387836.2007.02450.x.

21. Khorana AA, Francis CW, Culakova E, et al. Frequency, risk factors, and trends for venous thromboembolism among hospitalized cancer patients. Cancer. 2007;110:2339-2346. doi: 10.1002/cncr.23062.

22. Khorana AA, Francis CW, Culakova E, et al. Thromboembolism in hospitalized neutropenic cancer patients. J Clin Oncology. 2006;24:484-490. doi: 10.1200/JCO.2005.03.8877.

23. Agnelli G, Bolis G, Capussotti L, et al. A clinical outcome-based prospective study on venous thromboembolism after cancer surgery: The @RISTOS project. Ann Surg. 2006;243:89-95. doi: 10.1097/01.sla.0000193959.44677.48.
24. Simanek R, Vormittag R, Ay C, et al. High platelet count associated with venous thromboembolism in cancer patients: results from the Vienna Cancer and Thrombosis Study (CATS). J Thromb Haemost. 2010;8:114-120. doi: 10.1111/j.15387836.2009.03680.x.

25. Connolly GC, Khorana AA, Kuderer NM, et al. Leukocytosis, thrombosis and early mortality in cancer patients initiating chemotherapy. Thromb Res. 2010;126:113-118. doi: 10.1016/j. thromres.2010.05.012.

26. Dai H, Zhou H, Sun Y, et al. D-dimer as a potential clinical marker for predicting metastasis and progression in cancer. Biomed Rep. 2018;9:453-457. doi: 10.3892/br.2018.1151.

27. Sallah S, Husain A, Sigounas V, et al. Plasma coagulation markers in patients with solid tumors and venous thromboembolic disease receiving oral anticoagulation therapy. Clin Cancer Res. 2004;10:7238-7243. doi: 10.1158/1078-0432.CCR-04-0445.

28. Riondino S, Ferroni P, Zanzotto FM, et al. Predicting VTE in Cancer Patients: Candidate Biomarkers and Risk Assessment Models. Cancers (Basel). 2019;11:95. doi: 10.3390/ cancers11010095.

29. Arpaia G, Carpenedo M, Verga M, et al. D-dimer before chemotherapy might predict venous thromboembolism. Blood Coagul Fibrinolysis. 2009;20:170-175. doi: 10.1097/ MBC.ob013e32831bc2de.

30. Kondo S, Sasaki $\mathrm{M}$, Hosoi $\mathrm{H}$, et al. Incidence and risk factors for venous thromboembolism in patients with pretreated advanced pancreatic carcinoma. Oncotarget. 2018;9:1688316890. doi: 10.18632/oncotarget.24721.

31. Park K, Ryoo BY, Ryu MH, et al. Incidence of venous thromboembolism and the role of D-dimer as predictive marker in patients with advanced gastric cancer receiving chemotherapy: A prospective study. World J Gastrointest Oncol. 2017;9:176-183. doi: 10.4251/wjgo.v9.i4.176.

32. Wu J, Fu Z, Liu G, et al. Clinical significance of plasma D-dimer in ovarian cancer: A meta-analysis. Medicine. 2017;96:e7062. doi: 10.1097/MD.0000000000007062.

33. Ay C, Simanek R, Vormittag R, et al. High plasma levels of soluble P-selectin are predictive of venous thromboembolism in cancer patients: Results from the Vienna Cancer and Thrombosis Study (CATS). Blood. 2008;112:2703-2708. doi: 10.1182/blood-2008-02-142422.

34. Di Nisio M, Ferrante N, De Tursi M, et al. Incidental venous thromboembolism in ambulatory cancer patients receiving chemotherapy. Thromb Haemost. 2010;104:1049-1054. doi: 10.1160/TH10-05-0277.

35. Khorana AA, Dalal M, Lin J, et al. Incidence and predictors of venous thromboembolism (VTE) among ambulatory highrisk cancer patients undergoing chemotherapy in the United States. Cancer. 2013;119:648-655. doi: 10.1002/cncr.27772.

36. Numico G, Garrone O, Dongiovanni V, et al. Prospective evaluation of major vascular events in patients with nonsmall cell lung carcinoma treated with cisplatin and gemcitabine. Cancer. 2005;103:994-999. doi: 10.1002/cncr.20893.

37. Cunningham D, Starling N, Rao S, et al. Capecitabine and Oxaliplatin for Advanced Esophagogastric Cancer. N Engl J Med. 2008;358:36-46. doi: 10.1056/NEJMoa073149.

38. John AH. Epidemiology of venous thromboembolism. Nat Rev Cardiol. 2015;12:464-474. doi: 10.1038/nrcardio.2015.83.

39. Lee AYY, Levine MN, Butler G, et al. Incidence, risk factors, and outcomes of catheter-related thrombosis in adult patients with cancer. J Clin Oncol. 2006;24:1404-1408. doi: 10.1200/ JCO.2005.03.5600. 
40. Prandoni P, Lensing AWA, Piccioli A, et al. Recurrent venous thromboembolism and bleeding complications during anticoagulant treatment in patients with cancer and venous thrombosis. Blood. 2002;00:3484-3488. doi: 10.1182/ blood-2002-01-0108.

41. Lee AY, Levine MN, Baker RI, et al. Low-molecular-weight heparin versus a coumarin for the prevention of recurrent venous thromboembolism in patients with cancer. $\mathrm{N}$ Engl J Med. 2003;349:146-153. doi: 10.1056/NEJMoa025313.

42. Lee AYY, Kamphuisen PW, Meyer G, et al. Tinzaparin vs warfarin for treatment of acute venous thromboembolism in patients with active cancer: a randomized clinical trial. JAMA. 2015;314:677-686. doi: 10.1001/jama.2015.9243.

43. Kearon C, Akl EA, Ornelas J, et al. Antithrombotic therapy for VTE Disease: CHEST guideline and expert panel eport. Chest. 2016;149:315-352. doi: 10.1016/j.chest.2015.11.026.

44. Meyer G, Marjanovic Z, Valcke J, et al. Comparison of lowmolecular-weight heparin and warfarin for the secondary prevention of venous thromboembolism in patients with cancer: A randomized controlled study. Arch Intern Med. 2002;162:1729-1735. doi: 10.1001/archinte.162.15.1729.

45. Deitcher SR, Kessler CM, Merli G, et al. ONCENOX Investigators. Secondary prevention of venous thromboembolic events in patients with active cancer: enoxaparin alone versus initial enoxaparin followed by warfarin for a 180-day period. Clin Appl Thromb Hemost. 2006; 12:389-396. doi: 10.1177/1076029606293692.

46. Hull RD, Pineo GF, Brant RF, et al. Long-term low-molecularweight heparin versus usual care in proximal-vein thrombosis patients with cancer. Am J Med. 2006;119:1062-1072. doi: 10.1016/j.amjmed.2006.02.022.

47. Farge D, Bounameaux $H$, Brenner $B$, et al. International clinical practice guidelines including guidance for direct oral anticoagulants in the treatment and prophylaxis of venous thromboembolism in patients with cancer. Lancet Oncol. 2016;17:e452-e466. doi: 10.1016/S1470-2045(16)30369-2.

48. Farge D, Frere C, Connors JM, et al. International Initiative on Thrombosis and Cancer (ITAC) advisory panel. 2019 international clinical practice guidelines for the treatment and prophylaxis of venous thromboembolism in patients with cancer. Lancet Oncol. 2019;20:e566-e581. doi: 10.1016/S14702045(19)30336-5.

49. Hakoum MB, Kahale LA, Tsolakian IG, et al. Anticoagulation for the initial treatment of venous thromboembolism in people with cancer. Cochrane Database Syst Rev. 2018;1:CD006649. doi: 10.1002/14651858.CD006649.pub7.

50. Robertson L, Jones LE. Fixed dose subcutaneous low molecular weight heparins versus adjusted dose unfractionated heparin for the initial treatment of venous thromboembolism. Cochrane Database Syst Rev. 2017;2:CDo01100. doi: 10.1002/14651858. CD001100.pub4.

51. Key NS, Khorana AA, Kuderer NM, et al. Venous Thromboembolism Prophylaxis and Treatment in Patients With Cancer: ASCO Clinical Practice Guideline Update. J Clin Oncol. 2020;38:496-520. doi: 10.1200/JCO.19.01461.

52. Mazzolai L, Aboyans V, Ageno W, et al. Diagnosis and management of acute deep vein thrombosis: a joint consensus document from the European Society of Cardiology working groups of aorta and peripheral vascular diseases and pulmonary circulation and right ventricular function. Eur Heart J. 2018;39:4208-4218. doi: 10.1093/eurheartj/ehx003.
53. Raskob GE, van Es N, Verhamme P, et al. Edoxaban for the treatment of cancer-associated venous thromboembolism. N Engl J Med. 2018;378:615-624. doi: 10.1056/NEJMoa1711948.

54. Young AM, Marshall A, Thirlwall J, et al: Comparison of an oral factor Xa inhibitor with low molecular weight heparin in patients with cancer with venous thromboembolism: Results of a randomized trial (SELECT-D). J Clin Oncol. 2018;36:20172023. doi: 10.1200/JCO.2018.78.8034.

55. Li A, Garcia DA, Lyman GH, et al. Direct oral anticoagulant (DOAC) versus low-molecular-weight heparin (LMWH) for treatment of cancer associated thrombosis (CAT): A systematic review and meta-analysis. Thromb Res. 2019;173:158-163. doi: 10.1016/j.thromres.2018.02.144.

56. Hesketh PJ, Kris MG, Basch E, et al. Antiemetics: American Society of Clinical Oncology clinical practice guideline update. J Clin Oncol. 2017;35:3240-3261. doi: 10.1200/JCO.2017.74.4789.

57. Muñoz Martín AJ, Gallardo Díaz E, García Escobar I, et al. SEOM clinical guideline of venous thromboembolism (VTE) and cancer Clin Transl Oncol. 2020;22:171-186. doi: 10.1007/ S12094-019-02263-Z.

58. Francis CW, Kessler CM, Goldhaber SZ, et al. Treatment of venous thromboembolism in cancer patients with dalteparin for up to 12 months: The DALTECAN study. J Thromb Haemost. 2015;13:1028-1035. doi: 10.1111/jth.12923.

59. Jara-Palomares L, Solier-Lopez A, Elias-Hernandez T, et al. Tinzaparin in cancer associated thrombosis beyond 6months: TiCAT study. Thromb Res. 2017;157:90-96. doi: 10.1016/j. thromres.

60. Zurawska U, Parasuraman S, Goldhaber SZ. Prevention of pulmonary embolism in general surgery patients. Circulation. 2007;115:e302-e307. doi: 10.1161/CIRCULATIONAHA.

61. Bergqvist D, Agnelli G, Cohen AT, et al. Duration of prophylaxis against venous thromboembolism with enoxaparin after surgery for cancer. N Engl J Med. 2002;346:975-980. doi: 10.1056/NEJMoa012385.

62. Bustos Merlo AB, Arcelus Martınez JI, Turiño Luque JD, et al. Form of presentation, natural history and course of postoperative venous thromboembolism in patients operated on for pelvic and abdominal cancer. Analysis of the RIETE registry. Cir Esp. 2017;95:328-334. doi: 10.1016/j. ciresp.2017.05.006.

63. Fagarasanu A, Alotaibi GS, Hrimiuc R, et al. Role of extended thromboprophylaxis after abdominal and pelvic surgery in cancer patients: a systematic review and meta-analysis. Ann Surg Oncol. 2016;23:1422-1430. doi: 10.1245/s10434-0165127-1.

64. Di Nisio M, Porreca E, Candeloro M, et al. Primary prophylaxis for venous thromboembolism in ambulatory cancer patients receiving chemotherapy. Cochrane Database Syst Rev. 2016;12:CD008500. doi: 10.1002/14651858.CD008500.pub4.

65. Khorana AA, Francis CW, Kuderer NM, et al. Dalteparin thromboprophylaxis in cancer patients at high risk for venous thromboembolism: a randomized trial. Thromb Res. 2017;151:89-95. doi: 10.1016/j.thromres.2017.01.009.

66. Khorana AA, Soff GA, Kakkar AK, et al. Rivaroxaban for thromboprophylaxis in high-risk ambulatory patients with cancer. N Engl J Med. 2019;380:720-728. doi: 10.1056/ NEJMoa1814630.

67. Carrier M, Abou-Nassar K, Mallick R, et al. Apixaban to prevent venous thromboembolism in patients with cancer. $\mathrm{N}$ Engl J Med. 2019;380:711-719. doi: 10.1056/NEJMoa1814468. 
68. Verso M, Agnelli G. Venous thromboembolism associated with long-term use of central venous catheters in cancer patients. J Clin Oncol. 2003;21:3665-3675. doi: 10.1200/JCO.2003.08.008.

69. Kahale LA, Tsolakian IG, Hakoum MB, et al. Anticoagulation for people with cancer and central venous catheters. Cochrane Database Syst Rev. 2018;6:CD006468. doi: 10.1002/14651858. CDo06468.pub6.

70. Lee AY, Levine MN, Butler G, et al. Incidence, risk factors, and outcomes of catheter-related thrombosis in adult patients with cancer. J Clin Oncol. 2006;24:1404-1408. doi: 10.1200/ JCO.2005.03.5600.

71. Saber W, Moua T, Williams EC, et al. Risk factors of catheterrelated thrombosis (CRT) in cancer patients: a patient-level data (IPD) meta-analysis of clinical trials and prospective studies. J Thromb Haemost. 2011;9:312-319. doi: 10.1111/j.15387836.2010.04126.x.

72. Kovacs MJ, Kahn SR, Rodger M, et al. A pilot study of central venous catheter survival in cancer patients using lowmolecular-weight heparin (dalteparin) and warfarin without catheter removal for the treatment of upper extremity deep vein thrombosis (the Catheter study). J Thromb Haemost. 2007;5:1650-1653. doi: 10.1111/j.1538-7836.2007.02613.x.

73. Kreuziger LB, Ageno W, Lee A. Management of incidental splanchnic vein thrombosis in cancer patients. Hematology Am Soc Hematol Educ Program. 2014;2014:318-320. doi: 10.1182/asheducation-2014.1.318.

74. Ihaddadene R, Le Gal G, Delluc A, et al. Dose escalation of low molecular weight heparin in patients with recurrent cancerassociated thrombosis. Thromb Res. 2014;134:93-95. doi: 10.1016/j.thromres.2014.04.028.

75. Carrier M, Le Gal G, Cho R, et al. Dose escalation of low molecular weight heparin to manage recurrent venous thromboembolic events despite systemic anticoagulation in cancer patients. J Thromb Haemost. 2009;7:760-765. doi: 10.1111/j.1538-7836.2009.03326.x.

76. Samuelson Bannow BR, Lee AYY, Khorana AA, et al. Management of anticoagulation for cancer-associated thrombosis in patients with thrombocytopenia: a systematic review. Res Pract Thromb Haemost. 2018;2:664-669. doi: 10.1002/rth2.12111. 